\title{
Lista de Ulvophyceae do estado de São Paulo
}

\author{
Diclá Pupo $^{1,2}$ \& Ana Carolina Saraiva Pena Coto $^{1}$ \\ ${ }^{1}$ Núcleo de Pesquisa em Ficologia, Centro de Pesquisa em Plantas Avasculares e Fungos, \\ Instituto de Botânica, SMA, CP 68041, 04045-972, São Paulo, SP, Brasil \\ ${ }^{2}$ Autor para correspondência: Diclá Pupo,e-mail: dpupoibot@yahoo.com.br
}

PUPO, D. \& COTO, A.C.S.P. Checklist of Ulvophyceae of the São Paulo State. Biota Neotrop. 11(1a): http://www. biotaneotropica.org.br/v11n1a/en/abstract?inventory+bn0091101a2011

Abstract: The checklist comprises an inventory achieved from 1999 by BIOTA/FAPESP Program, comparing with those realized from 1950. The list was also complemented with data from the State Herbarium Algae Collection (SP). The number of benthic marine chlorophytes mentioned for the State is 70 species, besides some in level of genera. From 1990 to 2010 the number of registered taxa was 60, some in level of genera. New citations for the State are 17 species, while other nine were no more mentioned at the last decade.

Keywords: Ulvophyceae, biodiversity of the State of São Paulo, BIOTA/FAPESP Program.

Number of species: In the world:890, in Brazil: 210, estimated in São Paulo State: 80, known in São Paulo State: 70.

PUPO, D. \& COTO, A.C.S.P. Lista das Ulvophyceae do Estado de São Paulo. Biota Neotrop. 11(1a): http://www. biotaneotropica.org.br/v11n1a/pt/abstract?inventory+bn0091101a2011

Resumo: A presente lista tem como base levantamento realizado a partir de 1999 pelo Programa BIOTA/FAPESP e consequiente comparação com inventários realizados desde a década de 1950. A lista também é complementada com registros da Coleção de Algas do Herbário do Estado (SP), do Instituto de Botânica, que incluem espécimes coletados desde o início do século passado. O número total de clorófitas marinhas bentônicas mencionadas para o Estado é de 70 espécies, além de alguns táxons não identificados em nível infragenérico. De 1990 até 2010 o número de táxons registrados foi de 60, sendo alguns apenas em nível de gênero. As novas citações para o Estado somam 17 espécies, enquanto outras nove deixaram de ser citadas na última década.

Palavras-chave: Ulvophyceae, biota paulista, Programa BIOTA/FAPESP.

Número de espécies: No mundo: 890, no Brasil: 200, estimadas no estado de São Paulo: 80, conhecidas no estado de São Paulo: 70. 


\section{Introdução}

A divisão Chlorophyta engloba inúmeras classes que apresentam grande diversidade no nível de organização do talo e no histórico de vida. Alguns autores, como Hoek et al. (1997), consideram as clorófitas distribuídas em onze classes, sendo quatro relativas à algas marinhas bentônicas (Bryopsidophyceae, Cladophorophyceae, Dasycladophyceae e Ulvophyceae). Esta repartição é fundamentada, além de características de ultraestrutura, a partir de um organismo unicelular primitivo que deu origem a várias linhagens paralelamente, resultando na ocorrência de um mesmo nível de organização do talo em diferentes classes. Contudo, seguindo Stewart \& Mattox (1978), as clorófitas bentônicas marinhas têm sido mais amplamente aceitas como sendo de uma única classe, Ulvophyceae (Wynne 2005), ao lado de outras que abrigam as demais algas verdes.

O conceito de Ulvophyceae, segundo Stewart \& Mattox (1978), baseia-se na estrutura do aparato flagelar das células reprodutivas móveis, no envoltório das células flageladas e na divisão celular. As células móveis apresentam um a dois pares de flagelos, sem mastigonemas. Os corpos basais apresentam quatro raízes microtubulares arranjadas cruciadamente e raízes menores, de dois tamanhos diferentes, que se alternam entre dois ou mais microtúbulos. Os flagelos apresentam ainda escamas e rizoplastos. A parede celular pode ser ou não calcificada e a divisão celular ocorre por meio de um sulco, com presença do fuso mitótico cêntrico e persistente e ausência de ficoplasto.

A classe engloba talos ramificados ou não, filamentosos, mono- e distromáticos, ou ainda tubulares, os quais são, por vezes, compactados formando estruturas de consistência esponjosa. Os talos podem apresentar estrutura celular uninucleada, multinucleada ou serem totalmente sifonáceos. O histórico de vida observado no grupo é o diplobiôntico, iso- ou heteromórfico (Guiry \& Guiry 2011). São incluídas na classe sete ordens, das quais, na flora do estado de São Paulo, temos representantes de Ulvales, Cladophorales, Bryopsidales e Dasycladales (Coto \& Pupo 2009).

\section{Metodologia}

A elaboração da presente lista partiu da publicação de Coto \& Pupo (2009) que representa uma síntese para as clorófitas marinhas bentônicas dentro do projeto "Flora Ficológica do estado de São Paulo" (BIOTA/FAPESP). O trabalho foi objeto da dissertação de mestrado de Coto (2007) que consistiu de um levantamento da flora e comparação desta com os estudos pioneiros de A. B. Joly para a Baía de Santos (Joly 1957) e o Litoral Norte (Joly 1965), do Litoral Sul de Ugadim (1973) e do estudo de Ulvales para o estado, de Kanagawa (1983), além de estudos pontuais, tais como Oliveira Filho e Berchez (1978), Berchez \& Oliveira (1992), Braga et al. (1997), Qi (1999) e Horta (2000). Os dados acima também foram complementados com trabalho posterior de Rocha-Jorge (2010), o qual realizou levantamento das macroalgas do Parque Estadual da Laje de Santos, pelo mesmo projeto do BIOTA. Por fim, a lista foi complementada com a Coleção de Algas do Herbário do Estado "Maria Eneyda P. Kaufmann Fidalgo" (SP), cujos registros incluem espécimes coletados desde o início do século passado.

\section{Resultados e Discussão}

\section{Lista de Ulvophyceae}

A seguinte sinopse dos táxons de algas verdes marinhas inclui todos aqueles já citados para o estado de São Paulo. Os táxons que não ocorreram em citações recentes (de 2000 a 2010) foram assinaladas com um asterisco (*) e aqueles que constam da Coleção de Algas do Herbário Maria Eneida P. K. Fidalgo, mas não estão citados em nenhuma publicação, foram assinaladas com dois (**), como se segue. Ordem Ulvales

FAMÍLIA CTENOCLADACEAE Bolbocoleon jolyi Yamaguishi-Tomita

FAMÍLIA GAYRALIACEAE Gayralia oxysperma (Kützing) K.L. Vinogradova ex Scagel et al. Protomonostroma sp.

FAMÍLIA ULVACEAE Ulva chaetomorphoides (Børgesen) Hayden, Blomster, Maggs, P.C.Silva, M.J.Stanhope \& J.R.Waaland* Ulva clathrata (Roth) C. Agardh*

Ulva compressa Linnaeus

Ulva fasciata Delile

Ulva flexuosa Wulfen subesp. flexuosa

Ulva lactuca Linnaeus

Ulva linza Linnaeus

Ulva micrococca (Kützing) Gobi*

Ulva paradoxa C. Agardh

Ulva prolifera O.F. Müller

Ulva rigida C. Agardh

FAMÍLIA ULVELLACEAE

Entocladia viridis Reinke

Pringsheimiella scutata (Reinke) Höhnel ex Marchewianka Pseudodendoclonium submarinum Wille

Stromatella monostromatica (P. Dangeard) Kornmann \& P. Sahling

Ordem Cladophorales

\section{FAMÍLIA CLADOPHORACEAE}

Chaetomorpha aerea (Dyllwyn) Kützing

Chaetomorpha antennina (Bory) Kützing

Chaetomorpha brachygona Harvey

Chaetomorpha gracilis (Kützing) Kützing

Chaetomorpha linum (O.F. Müller) Kützing**

Chaetomorpha minima F.S.Collins \& Hervey*

Chaetomorpha spiralis Okamura

Cladophora albida (Nees) Kützing

Cladophora brasiliana G. Martens

Cladophora catenata (Linnaeus) Kützing

Cladophora coelothrix Kützing

Cladophora corallicola Børgesen

Cladophora crispula Kützing**

Cladophora dalmatica Kützing

Cladophora lehmanniana (Lindenberg) Kützing

Cladophora montagneana Kützing

Cladophora pellucidoidea C. Hoek

Cladophora prolifera (Roth) Kützing

Cladophora rupestris (Linnaeus) Kützing

Cladophora sericea (Hudson) Kützing

Cladophora submarina P.L. Crouan \& H.M. Crouan*

Cladophora vagabunda (Linnaeus) C. Hoek

Cladophora spp.

Rhizoclonium africanum Kützing

Rhizoclonium hierogliphicum (C. Agardh) Kützing**

Rhizoclonium riparium (Roth) Kützing ex Harvey

FAMÍLIA SIPHONOCLADACEAE

Cladophoropsis membranacea (C. Agardh) Børgesen

FAMÍLIA VALONIACEAE

Ernodesmis verticillata (Kützing) Børgesen

Valonia macrophysa Kützing 
Valonia utricularis (Roth) C.Agardh

Ordem Bryopsidales

\section{FAMÍLIA BRYOPSIDACEAE}

Bryopsis corymbosa J. Agardh

Bryopsis hypnoides J.V. Lamouroux

Bryopsis indica A. Gepp \& A.S. Gepp*

Bryopsis pennata J.V. Lamouroux

Bryopsis plumosa (Hudson) C. Agardh

Bryopsis sp.

Derbesia marina (Lyngbye) Solier

Derbesia sp.

FAMÍLIA CODIACEAE

Codium decorticatum (Woodward) M. Howe*

Codium intertextum Collins \& Hervey

Codium isthmocladum Vickers

Codium spongiosum Harvey

Codium taylorii P.C. Silva

\section{FAMÍLIA CAULERPACEAE}

Caulerpa fastigiata Montagne

Caulerpa racemosa (Forsskål) J. Agardh var. racemosa

Caulerpa racemosa (Forsskål) J. Agardh var. occidentalis

(J. Agardh) Børgesen

Caulerpa racemosa (Forsskål) J. Agardh var. peltata (J.V. Lamouroux) Eubank*

Caulerpa sertularioides (S.G. Gmelin) M. Howe

Caulerpella ambigua (Okamura) Prud'homme \& Lockhorst

FAMÍLIA HALIMEDACEAE

Halimeda gracilis Harvey ex J. Agardh

Halimeda sp.

FAMÍLIA UDOTEACEAE

Avrainvillea elliottii A. Gepp \& E.S. Gepp*

Avrainvillea nigricans Decaisne

Boodleopsis pusilla (Collins) W.R. Taylor, A.B. Joly \&

Bernatowicz

Ordem Dasycladales

\section{FAMÍLIA POLYPHYSACEAE}

Acetabularia calyculus J.V. Lamouroux

Acetabularia crenulata J.V. Lamouroux

Acetabularia schenkii K. Möbius*

\section{Comentários}

A presente lista com dados de herbários não está completa, uma vez que não inclui a rica coleção ficológica do Herbário do Departamento de Botânica, Instituto de Biociências da USP (SPF), que de 1950 a 1970 teve à frente o Prof. A. B. Joly, tendo continuidade por seus sucessores. Este acervo deverá ser objeto de consulta em outra ocasião, a fim de complementar as informações existentes em coleções de algas no estado (Splink 2011).

\section{Comparação da flora com outras regiões do Brasil e características dos ecossistemas costeiros marinhos no Estado}

A flora das clorófitas marinhas no litoral paulista apresenta um evidente empobrecimento na riqueza das espécies, se comparada às da região do Nordeste do Brasil, uma vez que as características climáticas tropicais e a diversidade de habitats dos recifes conferem uma possibilidade maior para as algas verdes bentônicas se desenvolverem. Como descreve Oliveira Filho (1977), a região tropical da costa brasileira abriga uma flora muito rica, onde se destacam, dentre as verdes, as Siphonocladales, Codiales e Caulerpales. O estado de São Paulo está inserido na região temperada quente, correspondente às regiões Sul e Sudeste (Horta et al. 2001) e tem como característica uma perda significativa dos representantes de clorófitas, em detrimento de outros grupos de algas.

As Ulvophyceae crescem ao longo do litoral paulista tanto em costões rochosos, onde são mais abundantes, como em manguezais, onde colonizam habitats com grande variação de salinidade. A costa do Estado, influenciada pela sua conformação geomorfológica, apresenta diversas particularidades regionais. Ao norte, a Serra do Mar entra em contato quase contínuo com o mar, desde a Boracéia até o limite com o Rio de Janeiro. Neste trecho, as escarpas rochosas se estendem em direção ao mar formando baías e enseadas entrecortadas por ecossistemas típicos de costões rochosos. Em direção ao sul, na Baixada Santista, a Serra do Mar possibilita a formação de planícies descontínuas, recortadas por um sistema denso de rios e canais de maré na região de Santos, São Vicente e Guarujá. As áreas planas dos estuários formam manguezais de extrema fragilidade. Na região Sul, de Itanhaém até Cananéia, predominam as planícies com influência da oscilação das marés e abrigadas da ação direta das ondas. O transporte de grande quantidade de sedimentos à costa é feito pelos rios que cortam as planícies. Os manguezais formados nos estuários são os maiores e mais preservados do litoral paulista (São Paulo 1996).

\section{Comparação da flora atual com dados pretéritos}

O levantamento da flora das ulvofíceas marinhas de Coto (2007) foi realizado como parte do projeto "Flora Ficológica do estado de São Paulo" (BIOTA), cujas coletas tiveram início a partir de 1999. Este trabalho atualizou o conhecimento do grupo para o litoral paulista (Coto \& Pupo 2009), possibilitando compará-lo com dados pretéritos. Após este levantamento e incluindo várias outras citações deste período (Braga et al. 1997, Horta 2000, Barata \& Fujii 2006, Pereira 2007, Rocha-Jorge 2010), o número total de clorófitas marinhas bentônicas mencionadas para o Estado chegou a 70 espécies (além de vários táxons não identificados em nível infragenérico). E o número de táxons registrados do final da década de 1990 até o presente foi de 60, sendo alguns apenas em nível de gênero.

Dentre as espécies citadas, algumas são novas ocorrências para o litoral paulista e outras não foram mais encontradas na natureza, apesar da intensa busca em coletas no campo. Por outro lado, algumas espécies apenas caíram em sinonímia, uma vez que nos últimos anos grandes mudanças ocorreram na taxonomia do grupo (Hayden et al. 2003, Hayden \& Waaland 2004, Wynne 2005).

Na ordem Ulvales 18 espécies são mencionadas para o Estado, além de um táxon citado apenas em nível de gênero (Protomonostroma sp.). Dentre estas, quatro representam novas ocorrências recentes, todas epífitas sobre outras algas: Bolbocoleon jolyi, Pringsheimiella scutata, Stromatella monostromatica e Pringsheimiella scutata (Coto 2007, Barata \& Fujii 2006, Coto \& Pupo 2009). Outras três espécies deixaram de ser registradas na última década: Ulva chaetomorphoides (como Enteromorpha chaetomorphoides: Joly 1957, Oliveira Filho \& Berchez 1978, Kanagawa 1983), Ulva clathrata (como Enteromorpha clathrata: Joly 1965, Kanagawa 1983, Yaobin 1999; como Enteromorpha crinita: Kanagawa 1983) e Ulva micrococca (como Enteromorpha micrococca: Ugadim 1973). Uma quarta espécie, Gayralia oxysperma (como Monostroma oxyspermum: Joly 1957, 1965, Berchez \& Oliveira 1992, Qi 1999 e como Ulvaria oxysperma: Braga 1997, Braga et al. 1997), apesar de não ter sido encontrada por Coto (2007) e, portanto, estar fora da listagem de Coto \& Pupo (2009), provavelmente está presente na área estuarina da Ilha do Cardoso, Cananéia. Esta espécie coexiste no mesmo habitat de 
Protomonostroma sp., mas em bem menor densidade (Braga 1997), sendo confundida com esta na natureza.

Para a ordem Cladophorales são registradas 27 espécies, além de várias Cladophoras citadas apenas em nível de gênero (duas por Joly 1965, uma por Oliveira \& Berchez 1978, duas por Qi 1999 e uma por Coto \& Pupo 2009), por se tratar de um grupo de difícil delimitação morfológica. Dez espécies são novas ocorrências recentes: Chaetomorpha spiralis (Pereira 2007), Cladophora albida, C. brasiliana, C. catenata, C. corallicola, C. pellucidoidea e $C$. sericea (Coto 2007, Coto \& Pupo 2009), além de Ernodesmis verticillata (Horta 2000, Rocha-Jorge 2010), Valonia macrophysa (RochaJorge 2010) e Valonia utricularis (Horta 2000) que representam esforços recentes em estudos do infralitoral, tanto do continente quanto de ilhas e lajes. Duas espécies não foram mais registradas: Chaetomorpha mínima e Cladophora submarina, ambas no Litoral Sul (Ugadim 1973).

A ordem Bryopsidales tem 19 espécies registradas para o litoral paulista, além de três táxons citados apenas em nível de gênero: Bryopsis sp. (Joly 1957), Derbesia sp. (Joly 1957, 1965) e Halimeda sp. (Pereira 2007). Três espécies representam novas ocorrências recentes: Bryopsis corymbosa (Pereira 2007), B. hypnoides (Coto \& Pupo 2009), Codium spongiosum (Horta 2000). E das espécies descritas anteriormente, três não foram mencionadas na última década: Bryopsis indica (Joly 1957, Qi 1999), Codium decorticatum (Joly 1957, 1965, Oliveira Filho \& Berchez 1978) e Avrainvillea elliottii (como A. atlantica, Joly 1965, Joly et al. 1965a).

Barata (2008) menciona para o Litoral Norte, em sua revisão do gênero Caulerpa, C. racemosa var. peltata, mas não inclui material coletado recentemente, portanto não sabemos se esta variedade ainda ocorre nos locais citados. Por outro lado, confirmou-se para o Litoral Norte a ocorrência de Caulerpa sertularioides (Joly 1965, Coto \& Pupo 2009), Caulerpella ambigua (Coto \& Pupo 2009 e como Caulerpa vickersiae, segundo Joly 1965, Joly et al. 1965b) e Codium spongiosum (Horta 2000), confirmando ser este seu limite sul de ocorrência no litoral brasileiro (Santos 1983). Por outro lado, Codium isthmocladum, apesar de citado apenas para o Litoral Norte (Joly 1965, Horta 2000, Coto \& Pupo 2009), não deixa de ocorrer também na região sul do Brasil (Santos 1983).

Por fim, na ordem Dasycladales, das três espécies de Acetabularia, duas apresentam citações recentes: A. calyculus, para o Litoral Norte (Coto \& Pupo 2009) e A. crenulata, para o infralitoral de ilhas (Horta 2000), sendo que A. schenkii (Joly 1965) não foi mais encontrada.

\section{Principais Avanços ao Programa BIOTA/FAPESP}

Como já exposto acima, o projeto desenvolvido dentro do Programa BIOTA/FAPESP foi uma oportunidade importante que permitiu a atualização do conhecimento da flora de algas verdes bentônicas, onde se pode comparar dados atuais com dados pretéritos existentes. Também foi possível detectar as principais lacunas de conhecimento no grupo, o que é apontado em seguida.

\section{Principais Lacunas do Conhecimento}

Em relação aos levantamentos de flora, segundo Oliveira et al. (2002), o número de espécies de algas conhecidas no Brasil aumentaria no mínimo 20 a $30 \%$ se as amostragens fossem feitas no infralitoral. Apesar de ainda conhecermos relativamente poucas comunidades de infralitoral no Estado, principalmente nas ilhas, onde ocorrem bem menos estudos, no caso das algas verdes, a diversidade de espécies não seria tão maior significativamente do que a que se conhece atualmente. Isto porque as clorófitas bentônicas, com algumas exceções, predominam na região entremarés, devido às suas características fisiológicas, como o seu desempenho fotossintético, necessitando de maior luminosidade. Considere-se também que ao longo da costa paulista a água do mar apresenta bastante turbidez, se compararmos com outras regiões, como no nordeste brasileiro. A proximidade da Serra do Mar e a presença de estuários contribuem para esta turbidez da água, devido à presença de sedimentos próximo à costa.

No que diz respeito à taxonomia morfológica, esta tem se mostrado insuficiente para identificar espécies de certos gêneros das clorófitas. É o que ocorre, por exemplo, com as Ulvales, onde diferentes autores descrevem, para uma mesma espécie, diversas características morfológicas conflitantes, como o número de pirenóides, disposição das células no talo e presença de ramificações, devido à alta variabilidade fenotípica das espécies, provavelmente decorrente dos diferentes habitats onde ocorrem (Bliding 1968, Kanagawa 1983).

Atualmente, o cultivo em laboratório e a biologia molecular têm elucidado inúmeros problemas taxonômicos e sugerido novas circunscrições dos gêneros. Em Ulvaceae, os estudos moleculares e de cultura acarretaram mudanças importantes na delimitação do gênero Ulva, ao incorporar as espécies tubulares que anteriormente pertenciam a Enteromorpha (Hayden et al. 2003, Wynne 2005). Espécies de Ulva do litoral paulista, onde o gênero é bem representado, ainda necessitam de uma revisão criteriosa incluindo estudos moleculares, uma vez que o gênero possui complexos de espécies semelhantes morfologicamente, como é o caso de $U$. compressa e as diferentes variedades de $U$. clathrata.

O gênero Protomonostroma, da família Gayraliaceae, só pode ser identificado na flora local por meio de cultura em laboratório que elucidou a sua ontogenia, imprescindível para determinar os vários gêneros monostromáticos, tais como Monostroma, Ulvaria e Gayralia (Tanner 1981). E ainda falta identificar a espécie, uma vez que $P$. undulatum, citada na literatura, difere dos espécimes estudados.

As famílias que antes pertenciam a Chaetophoraceae ainda necessitam de estudos de revisão: Ulvellaceae, representada na flora paulista por Entocladia, Pringsheimiella e Stromatella e Ctenocladaceae, representada por Bolbocoleon. Para estes representantes microscópicos, a cultura em laboratório mostrou ser boa ferramenta para elucidar a ontogenia e morfologia do talo, assim como os seus históricos de vida (Kornmann \& Sahling 1983, O'Kelly \& Floyd 1983). Tanto que a identificação de Stromatella monostromatica (Barata \& Fujii 2006, Coto 2007) só foi possível a partir de desenvolvimentos em cultura.

As espécies de Cladophora também apresentam variações morfológicas significativas em função do habitat (Hoek 1963, 1982, Santos 1983, Coto \& Pupo 2009). Neste grupo, a biologia molecular representa ferramenta importante para a futura delimitação das espécies (Hoek \& Chihara 2000, Gestinari 2004). Os gêneros Chaetomorpha e Rhizoclonium também merecem um estudo de revisão com base em biologia molecular, uma vez que ao longo das últimas décadas, algumas espécies descritas para São Paulo e o Brasil foram colocadas em sinonímias, apesar de nítidas diferenças morfológicas observadas entre elas, como $R$. hookeri para $R$. africanum, $R$. kerneri para $R$. riparium (Wynne 1998, 2005), enquanto a espécie $R$. tortuosum passou para Chaetomorpha gracilis.

Além do aspecto taxonômico, ainda é preciso investigar os usos econômicos das algas verdes. Como matéria-prima, espécies de Ulva e de gêneros monostromáticos são cultivados para fins alimentícios, como condimentos ou corantes e na confecção de embalagens biodegradáveis (Ohno \& Rebello 1995a, 1995b). Na bioprospecção, diversos gêneros podem apresentar potencial biotecnológico. É o caso de Caulerpa racemosa, C. sertularioides e de Ulva lactuca que 
apresentam lectinas com atividades hemoaglutinantes (Sampaio et al. 1998, Freitas et al. 1997).

\section{Principais Grupos de Pesquisa em Ulvophyceae}

O Instituto de Botânica, da Secretaria de estado do Meio Ambiente possui um dos principais grupos de pesquisa em algas marinhas bentônicas no Estado. Ulvophyceae, em especial, tem sido objeto de estudos taxonômicos de Diclá Pupo, Mutue T. Fujii, Nair S. Yokoya e Sílvia M. P. B. Guimarães, incluindo estudos moleculares coordenados pela Dra. Mutue. Estudos em ecofisiologia, de histórico de vida e desenvolvimento em cultura são encabeçados pela Dra. Nair S. Yokoya.

O Departamento de Botânica da Universidade de São Paulo possui o grupo de pesquisa em algas marinhas bentônicas que foi pioneiro no Brasil, desde o Dr. Aylthon Brandão Joly. Conta atualmente com cinco pesquisadores nas mais variadas áreas do conhecimento em algas marinhas, sendo que alguns estudos moleculares têm sido realizados pela Dra. Mariana Cabral de Oliveira, em parceria com o Instituto de Botânica (Barata 2008).

\section{Principais Acervos}

- Coleção de Algas do Herbário do Estado "Maria Eneyda P. Kaufmann Fidalgo" (SP)

Curadora atual: Dra. Célia Leite Sant'Anna.

Instituto de Botânica, SMA

Centro de Pesquisa em Plantas Avasculares e Fungos

Núcleo de Pesquisa em Ficologia

Av. Miguel Estéfano 3687 - São Paulo, SP, CEP 04301-012

- Herbário do Departamento de Botânica (SPF)

Curadora atual: Dra. Valéria Cassano

Instituto de Biociências

Universidade de São Paulo

Rua do Matão 277, ed. Sobre-as-ondas - Cidade Universitária - São Paulo, SP, CEP 05508-090

\section{Perspectivas de Pesquisa do Grupo para os Próximos 10 anos}

No que concerne às algas verdes, será prioritário realizar revisões dos gêneros que apresentam problemas taxonômicos, como Ulva, Chaetomorpha, Cladophora e Rhizoclonium, incorporando estudos moleculares. O grupo de Ulvellaceae também necessita de mais estudos, envolvendo desenvolvimento em cultura de laboratório. O grupo de ficólogos do Instituto de Botânica também tem o potencial para realizar estudos de bioprospecção em algas verdes marinhas.

\section{Referências Bibliográficas}

AMADO FILHO, G.M., HORTA, P.A., BRASILEIRO, P.S., BARRETO, M.B.B.B. \& FUJII, M.T. 2006. Subtidalbenthic marine algaeofthe Marine State Park of Laje de Santos (São Paulo, Brazil). Braz. J. Oceanogr. 54:121. http://dx.doi.org/10.1590/S1679-87592006000300006

BARATA, D. \& FUJII, M.T. 2006. Ctenocladaceae e Ulvellaceae (Chlorophyta) do Espírito Santo e Litoral Norte de São Paulo, Brasil. Hoehnea33:359-370.

BARATA, D. 2008. Taxonomia e filogenia do gênero Caulerpa J.V. Lamour. (Bryopsidales, Chlorophyta) no Brasil. Tese de doutorado, Instituto de Botânica, São Paulo.

BERCHEZ, F.A.S. \& OLIVEIRA, E.C.1992. Temporal changes in the benthic marine flora of the Baía de Santos, SP, Brazil, over the last four decades. In Algae and Environment: a general approach. (M. Cordeiro-Marino, M.T.P. Azevedo, C.L. Sant'Anna, N. Yamaguishi-Tomita, \& E.M Plastino. eds.). Sociedade Brasileira de Ficologia, São Paulo, p.120-131.
BLIDING, C.1968. A criticalsurveyofEuropean taxa in Ulvales. Part II. Ulva, Ulvaria, Kornmannia. Bot. Not. 121:535-629.

BRAGA, M.R.A.1997. Recruitment of two species of monostromatic bladelike chlorophytes, Monostromasp. and Ulvariaoxysperma (Ulvales, Chlorophyta), in São Paulo, Brazil.Phycol. Res. 45:153-161.http://dx.doi. org/10.1111/j.1440-1835.1997.tb00088.x

BRAGA, M.R.A. FUJII, M.T. \& CORDEIRO-MARINO, M.1997. Monostromaticgreenalgae (Ulvales, Chlorophyta)of São Paulo and Paraná States (Brazil): distribution, growth and reproduction. Rev. Bras. Bot. 20:197-203.http://dx.doi.org/10.1590/S0100-84041997000200010

COTO, A.C.S.P. 2007. Biodiversidade de clorófitas marinhas bentônicas do litoral do estado de São Paulo. Dissertação de mestrado, Instituto de Botânica, São Paulo.

COTO, A.C.S.P. \& PUPO, D. 2009. Ulvophyceae. In Flora Ficológica do Estado de São Paulo (C.E.M. Bicudo, org.). FAPESP;Rima, São Carlos, v.3 p.1-76.

FREITAS, A.L.P., TEIXEIRA, D.I.A., COSTA, F.H.F., FARIAS, W.R.L., LOBATO, A.S.C., SAMPAIO, A.H., BENEVIDES, N.M.B.1997. A new survey of Brazilian marine algae for agglutinins. J. Appl. Phycol. 9:495-501.

GESTINARI, L.M.S. 2004. Taxonomia e distribuição do gênero CladophoraKützing (Cladophorales, Chlorophyta) no litoral brasileiro. Tese de doutorado, Universidade Federal Rural de Pernambuco, Recife.

GUIRY, M.D. \& GUIRY, G.M. 2011. AlgaeBase.World-wide electronic publication, National University of Ireland, Galway.http://www.algaebase. org (últimoacessoem 28/02/2011).

HAYDEN, H., BLOMSTER, J., MAGGS, C., SILVA, P., STANHOPE, M.J. \& WAALAND, J.R. 2003. Linnaeus was right all along: UlvaandEnteromorphaare not distinct genera. European J. Phycol. 38:277-294.http://dx.doi.org/10.1080/1364253031000136321

HAYDEN, H. \& WAALAND, J.R. 2004. A molecular systematic study of Ulva(Ulvaceae, Ulvales) from the northeast Pacific.Phycologia 43:364382. http://dx.doi.org/10.2216/i0031-8884-43-4-364.1

HOEK, C. van den. 1963. Revision of theeuropeanspecies of Cladophora. Brill, Leiden.

HOEK, C. van den. 1982. A taxonomicrevision of the American species of Cladophora (Chlorophyceae) in the North AtlanticOcean and theirgeographicdistribution. North-Holland Publishing Company, Amsterdam.

HOEK, C. van den \& CHIHARA, M. 2000. A taxonomicrevision of the marine species of Cladophora (Chlorophyta) alongthecoasts of Japan and theRussianfar East. Nacional ScienceMuseum, Tokyo.

HOEK, C. van den, MANN, D.G. \& JAHNS, H.M. 1997. Algae: anintroductiontophycology. Cambridge UniversityPress, Cambridge.

HORTA, P.A.2000. Macroalgas do infralitoral do sul e sudeste do Brasil: taxonomia e biogeografia. Tese de doutorado, Universidade de São Paulo, São Paulo.

HORTA, P.A., AMANCIO, E., COIMBRA, C.S. \& OLIVEIRA, E.C.2001. Considerações sobre a distribuição e origem da flora de macroalgas marinhas brasileiras. Hoehnea28:243-265.

JOLY, A.B.1957. Contribuição ao conhecimento da flora ficológica da Baía de Santos e arredores. Bol. Fac. Filos. Cienc. Let., Univ. São Paulo, Bot. 14:1-199.

JOLY, A.B.1965. Flora marinha do litoral norte do estado de São Paulo e regiões circunvizinhas. Bol. Fac. Filos. Cienc. Let., Univ. São Paulo, Bot. 21:1-267.

JOLY, A.B., CORDEIRO, M., YAMAGUISHI, N. \& UGADIM, Y. 1965a. New marine algae from southern Brazil.Rickia 2:159-182.

JOLY, A.B., CORDEIRO, M., YAMAGUISHI, N. \& UGADIM, Y. 1965b. Additions to the marine flora of Brazil IV.Rickia2:129-139.

KANAGAWA, A.I.1983. Ulvales (Chlorophyta) marinhas do estado de São Paulo, Brasil. Dissertação de mestrado, Universidade de São Paulo, São Paulo. 
KORNMANN, P. \& SAHLING, P.H. 1983. Meeresalgen von Helgoland: Ergänzung. Helgol.Wiss. Meeresunters. 36:1-65.http://dx.doi. org/10.1007/BF01995795

OHNO, M. \& REBELLO, J.1995a. Cultivo de Enteromorpha. In Manual de métodos ficológicos (K. Alveal, M. E. Ferrario, E. C. Oliveira y E. Sar, eds.) Universidad de Concepción, Concepción, p.513-520.

OHNO, M. \& REBELLO, J. 1995b. Cultivo de Monostroma. In Manual de métodos ficológicos (K. Alveal, M. E. Ferrario, E. C. Oliveira y E. Sar, eds) Universidad de Concepción, Concepción, p.521-527.

O'KELLY, C.J. \& FLOYD, G.L.1983.The flagellar apparatus of Entocladiaviridismotile cells, and the taxonomic position of the resurrected family Ulvellaceae (Ulvales, Chlorophyta).J. Phycol. 19:153164.http://dx.doi.org/10.1111/j.0022-3646.1983.00153.x

OLIVEIRA, E.C., HORTA P.A., AMANCIO C.E. \& SANT'ANNA, C.L. 2002. Algas e angiospermas marinhas bênticas do litoral brasileiro: diversidade, explotação e conservação. In Avaliação e ações prioritárias para a conservação da biodiversidade das zonas costeira e marinha. (Ministério do Meio Ambiente, ed.). Brasília. CDRom, FTT.

OLIVEIRA FILHO, E.C.1977. Algas marinhas bentônicas do Brasil. Tese de livre docência, Universidade de São Paulo, São Paulo.

OLIVEIRA FILHO, E.C. \& BERCHEZ, F.A.S.1978. Algas marinhas bentônicas da baía de Santos - alterações da flora no período de 19571978. Bol. Bot. 6:49-59.

PEREIRA, A.P.V. 2007. Caracterização taxonômica da comunidade marinha bentônica de substrato consolidado do infralitoral no costão oeste da Enseada de Palmas, Parque Estadual da Ilha Anchieta, Ubatuba, SP, Brasil. Tese de doutorado, Universidade de São Paulo, São Paulo.

QI, Y. 1999. Estudos sobre a variação temporal da composição de macroalgas marinhas em uma baía poluída - o caso de Santos, Litoral de São Paulo, Brasil. Tese de doutorado, Universidade de São Paulo, São Paulo.
ROCHA-JORGE, R. 2010. Diversidade de macroalgas do Parque Estadual Marinho da Laje de Santos, SP, Brasil. Dissertação de mestrado, Instituto de Botânica, São Paulo.

SAMPAIO, A.H., ROGERS, D.J., BARWELL, C.J. 1998. Isolation and characterization of the lectin from the green marine alga Ulvalactuca Linnaeus.Bot. Mar. 41:427-443.http://dx.doi.org/10.1515/ botm.1998.41.1-6.427

SANTOS, D.1983. Clorofíceas bentônicas marinhas do Estado de Santa Catarina. Dissertação de mestrado, Universidade de São Paulo, São Paulo.

SÃO PAULO (Estado). Secretaria de Estado do Meio Ambiente - SMA. 1996. Atlas das unidades de conservação ambiental do estado de São Paulo. Parte I. Litoral. Secretaria de Estado do Meio Ambiente, São Paulo.

SPLINK. Species Link. http://www.splink.org.br (último acesso em 25/02/2011).

STEWART, K.D. \& MATTOX, K.R.1978.Structural evolution in the flagellated cells of green algae and land plants.BioSystems 10:145-152. http://dx.doi.org/10.1016/0303-2647(78)90036-9

TANNER, C.E.1981. Chlorophyta: Life histories. In The biology of the seaweeds (C.S. Lobban\& M.J. Wynne eds.). Blackwell Scientific Publications, Oxford, p.218-247.

UGADIM, Y.1973. Algas marinhas bentônicas do litoral sul do Estado de São Paulo e do litoral do Estado do Paraná. I. Divisão Chlorophyta. Bol. Bot. 1:11-77.

WYNNE, M.J.1998. A checklist of benthic marine algae of the tropical and subtropical Western Atlantic: second revision. Beih.Nova Hedw. 116:1-155.

WYNNE, M.J. 2005. A checklist of benthic marine algae of the tropical and subtropical Western Atlantic: second revision. Beih. Nova Hedw. 129:1-152. 